\title{
Health Impacts and Characteristics of \\ Deprescribing Interventions in Older Adults - a Protocol of a Systematic Review and Meta-analysis
}

\section{Zoé Tremblay}

Universite Laval

Martine Marcotte

Centre d'excellence sur le vieillissement de Québec

David Mumbere-Bamusemba

Universite Laval

Danielle Laurin

Universite Laval

Daniela Furrer Soliz-Urrutia

Universite Laval

Lise Poisblaud

Centre d'excellence sur le vieillissement de Québec

\section{Barbara Farrell}

University of Ottawa

Caroline Sirois

Universite Laval

Anik MC Giguère

Universite Laval

André Tourigny

Universite Laval

Isabelle Vedel

McGill University

Pierre-Hugues Carmichael

Centre d'excellence sur le vieillissement de Québec

José Morais

McGill University

Edeltraut Kröger ( $\nabla$ edeltraut.kroger.ciussscn@ssss.gouv.qc.ca )

BPharm, MSc, PhD, Hôpital du Saint-Sacrement, 1050 Chemin Ste-Foy, room L2-30, Québec (QC), Canada https://orcid.org/0000-0002-5653-3979 
Keywords: Deprescribing, Older adult, Aging, Medication use, Inappropriate prescribing, Potentially inappropriate medication, Polypharmacy, Comorbidity, Multimorbidity, Systematic review

Posted Date: July 30th, 2020

DOI: https://doi.org/10.21203/rs.3.rs-50167/v1

License: (c) (1) This work is licensed under a Creative Commons Attribution 4.0 International License. Read Full License

Version of Record: A version of this preprint was published at JMIR Research Protocols on October 21st, 2020. See the published version at https://doi.org/10.2196/25200. 


\section{Abstract}

\section{Background}

Deprescribing, a relatively recent concept, has been proposed as a promising solution to the growing issues of polypharmacy and use of medications of questionable benefit among older adults. However, little is known about the health outcomes of deprescribing interventions. This study aims to contribute to the knowledge on deprescribing by adressing two specific objectives: 1) describe the impact of deprescribing in adults' $\geq 60$ years on health outcomes or quality of life; and 2) determine the characteristics of effective interventions in deprescribing.

Methods

Primary studies targeting three concepts (older adults, deprescribing, and health/quality of life outcomes) will be included in the review. The search will be performed using key international databases (MEDLINE, EMBASE, CINAHL, Ageline, Psyclnfo) and a special effort will be made to identify grey literature. Two reviewers will independently screen the articles, extract the information and evaluate the quality of the selected studies. If methodologically feasible, meta-analyses will be performed for groups of intervention studies reporting on deprescribing interventions for similar medications, used for similar or identical indications and reporting on similar outcomes (for example benzodiazepines used against insomnia and reporting on quality of sleep or quality of life). Alternatively, results will be presented in bottom-line statements (Objectives 1) and a matrix outlining effective interventions (Objective 2).

Discussion

The knowledge synthesis may be limited by the availability of high-quality, clinical trials on deprescribing and its outcomes in older adults. Additionally, analyses will likely be affected by studies on the deprescribing of different types of molecules within the same indication, e.g. different pharmacological classes and medications to treat hypertension, and different measures of health and quality of life outcomes for the same indication.

Systematic Review Registration Number: CRD42015020866

\section{Background}

\section{Polypharmacy and older adults}

The use of multiple medications in older adults is on the rise (1), and the well-known risk of adverse medication events, defined as the undesired effects or toxicities caused by a medication, increases with the number of medications prescribed (2). Polypharmacy (i.e. $>5$ concurrent medications) among older adults is a worldwide problem with prevalence estimates varying between $39 \%$ for the USA in 2010 (3) to $74 \%$, over 12 years in Sweden in 2018 (4). Associated with aging is the onset of chronic conditions, often co-existing in a phenomenon termed multimorbidity (2). Polypharmacy may be deemed necessary, or 
rational, for patients suffering from multimorbidity, since, in many cases, at least one medication is needed to treat each condition (6). Polypharmacy is, however, associated with many adverse outcomes, including undesired medication reactions, increased time of hospital stay, risk of readmission to hospital soon after discharge, falls, and mortality (2). The risk of an adverse medication event has been estimated at $13 \%$ with two medications, increasing to $58 \%$ and $82 \%$ with five and seven or more medications, respectively (7), underlining the need to limit medication use and to prescribe wisely among older, and particularly more vulnerable adults.

\section{Inappropriate prescribing}

Pharmacotherapy is deemed inappropriate when the risks associated with the used medications exceed its benefits (6). This risk/benefit ratio should therefore be considered by health professionals before prescribing a medication, and it often differs for seniors as they encounter a physiological decline in all aspects of pharmacodynamics and pharmacokinetics (8). Indeed, potentially inappropriate medication (PIM) can be dangerous for older patients (9). Several lists of potentially inappropriate medications (PIMs) have been elaborated in an attempt to limit inappropriate prescribing. Explicit criteria, such as the Beers criteria from the United States (10), or the STOPP/START criteria from Europe (11), are widely known and have been adapted to the clinical context of specific countries (12-14). Health professionals may also use implicit criteria, such as the Medication Appropriateness Index (15). Given the great number of PIMs and their consequences, medication reviews should be conducted on a regular basis, especially for older adults (6) but health care providers still struggle with this process (16).

\section{Interventions to improve inappropriate prescribing and polypharmacy}

In recent years, interventions to decrease inappropriate prescribing and polypharmacy have increasingly been developed. A Cochrane review (17) and its updates (16), (18) studied such interventions: results from randomized controlled trials (RCT) on interventions (educational programs for prescribers, medication reviews by pharmacists and tools to aid in clinical decision making), showed effectiveness in decreasing inappropriate prescribing, but their clinical effects remained unclear (18).

\section{Deprescribing}

The deprescribing process is defined as the reduction, tapering or discontinuation of medication deemed inappropriate for a specific patient, with the aim of minimizing polypharmacy and improving patient outcomes (19). Presently, there is a lack of knowledge regarding the association of specific, effective elements of deprescribing for certain medication classes and resulting health outcomes. Indeed, knowledge users $(\mathrm{KU})$, participating in the development of this study, i.e. the National Stakeholder Council on Safe Medication Management for Older Men and Women of the Canadian Institutes of Health research (http://www.cihr-irsc.gc.ca/e/49001.html), physicians, pharmacists, and patients expressed the need for evidence on health outcomes and quality of life, for deprescribing. 
Findings from some RCT are encouraging: deprescribing of antipsychotics was found to have no detrimental effects $(20,21)$, and to reduce the risk of falls $(22)$. Deprescribing of benzodiazepines showed subtle cognitive advantages (23), while the discontinuation or dose-reduction of statins in patients with reduced life expectancy had no negative impact (24). As for the deprescribing of chronic diuretics, one study reported preserved health outcomes after deprescribing $(26)$, but two others failed to do so $(25,27)$. Page and al.'s 2016 review aimed to determine if deprescribing is a safe, effective and feasible intervention to reduce mortality in older adults. Their systematic review reporting on non-randomized deprescribing interventions, showed a significant decrease in mortality: it found that generalized education programs had no impact on mortality, but patient-specific interventions decreased mortality (35). Also, systematic reviews on the effects of deprescribing of specific medication classes have been performed in past years e.g. for proton pump inhibitors, benzodiazepines and antipsychotics (28-30) in order to develop algorithms to guide deprescribing in clinical practice. Nevertheless, challenges remain regarding the evidence on the health effects of deprescribing interventions for numerous medication classes and older adults in particular. To fill this knowledge gap, the present systematic review will include all recently published intervention studies and report on the characteristics of interventions successful at reducing medication and at improving or maintaining older adults' health or quality of life. Ultimately, this systematic review aims to contribute to the development of an interdisciplinary consensus on effective interventions in deprescribing, which may lead to the development of guidelines useful for health professionals and patients.

\section{Objectives}

More specifically, the review will describe the impact on health and quality of life of deprescribing in patients aged 60 years and older as well as the characteristics of effective deprecribing interventions. To meet these objectives, the study aims to answer the following research questions:

1. What are the impacts of deprescribing interventions in older adults on health outcomes or quality of life?

2. What are the characteristics of deprescribing interventions, or elements thereof, that achieve positive or at least neutral effects on the health or quality of life of older adults?

\section{Methods}

\section{Literature search}

The review method will be based on the Cochrane Handbook for Systematic Reviews of Interventions (31) and the Methods for the development of public health guidance of the National Institute for Health and Care Excellence (32). The following key words and terms will be combined to identify deprescribing intervention studies:

a. polypharmacy, deprescribing, Beers's criteria, potentially inappropriate

b. withdraw*, withhold, withheld, stop*, cease*, discontinu*, reduc* 


\section{c. aged, geriatri*, frai*}

$\mathrm{KU}$ will advise on relevant key papers to inform the development of search strategies by two scientific librarians in consultation with the review authors. These strategies will be adapted for each database. References will be searched using key international databases such as MEDLINE, EMBASE, CINAHL, Ageline and Psyclnfo without date limitation. When available, limits will be set to restrict the search to humans aged 60 and over and publications in English, French or German but without time limits. The search strategy is featured in Appendix 1.

Reference lists of relevant review articles and of included studies will be checked manually for additional relevant articles. Authors will make a special effort to identify relevant grey literature. See Appendix 2 for the probable, but not exhaustive, list of databases and websites.

\section{Study selection}

Study selection will be performed by two independent reviewers according to the following inclusion and exclusion criteria (PICOS):

- Population studied: Study groups with participants aged 60 years and older will be considered for this review. Study groups with a mean age $\geq 60$ years, for which at least $80 \%$ of participants were $\geq$ 60 years old, or offering the possibility to extract data related to a subgroup of participants aged $\geq$ 60 years. In addition, the participants will have at least one medication prescribed for a chronic condition.

- Interventions: Deprescribing interventions, regardless of the intervention target (patients, caregivers or health professionals) in any intervention setting (hospital, nursing home, etc.) will be selected.

- Comparison: Only the comparison of deprescribing interventions with usual care or different head-tohead interventions will be considered for this review.

- Outcome: For interventions having affected the participants' medication regimen all health outcomes will be considered, including withdrawal symptoms, adverse medication reactions, clinical outcomes, cognition, behavior, falls, use of health services, quality of life, mortality or survival.

- Study design: All robust study designs will be included (RCT, non-RCT; controlled before-after studies; interrupted time series studies; repeated measures).

All included studies will be primary studies, therefore reviews, editorials, letters to the editor, commentaries, and other similar publications will be excluded.

All identified references will be combined into an EndNote® library (https://endnote.com/) and multiple copies will be eliminated. The systematic review software DistillerSR® (www.evidencepartners.com/) will be used for the subsequent steps. Two reviewers will independently determine the eligibility of the retrieved studies by comparing their titles and abstracts to the inclusion criteria. Subsequently, the full 
texts of the retained articles will be screened to confirm their relevance. The process will be similar for all types of literature sources. The study selection form can be found in Appendix 3.

\section{Data extraction}

The extraction of data will be completed using DistillerSR $B$ and pre-established forms regarding

- Study characteristics (design, date, and location)

- Population selection and participants' characteristics (age, sex, residency)

- Intervention description (providers, targets, duration, follow-up)

- Outcomes (medication regimen, health, quality of life)

An example of a data extraction form is featured in Appendix 4.

The Cochrane Collaboration's tool GRADE system for grading the quality of evidence will be used to evaluate the quality of the evidence. The risk of bias of eligible studies will be assessed using the SIGN tool for RCTs (33) and the Research Triangle Institute (RTI) instrument for non-randomized studies (34). The process will be carried out by one reviewer and reviewed by a second one.

\section{Data synthesis}

A meeting between researchers and stakeholders allowed to prioritize medication classes with specific indications (for example bisphosphonates against osteoporosis) over a specific health outcome (as for example mortality reduction as in the review by Page et al) (35). If methodologically feasible, metaanalyses will be performed for intervention studies reporting on deprescribing interventions for similar medications or a specific medication class, used for similar or identical indications and reporting on similar outcomes (for example benzodiazepines against insomnia, reporting on sleep quality). If metaanalyses are impossible, the study results will be summarized in a transparent and reproducible narrative synthesis, based on the methods published by Popay et al. (36) and by Rodgers et al. (37) (Objectives 1). Descriptive numerical summary tables will also be completed. In order to answer objective 2, we will identify the most effective interventions or intervention components in regard, but not limited to the following patient characteristics:

- Author, year, country

- Study design and setting

- Number of participants, Mean (SD) age, Male proportion (\%)

- Intervention/control

- Outcome measures

- Follow-up duration

- Study results

Effect of intervention on discontinuation 
Health outcomes, quality of life outcomes

Comparative qualitative analysis will be used to analyze the causal contribution of different intervention components towards health outcomes (38). The sets of characteristics associated with the specific outcome will be charted. Afterwards, they will be subjected to a minimization procedure to identify a simpler set of conditions accounting for observed health outcomes. This will result in a matrix of intervention characteristics and related outcomes.

\section{Meta-analyses}

If outcomes or medications are sufficiently similar, the results of deprescribing interventions will be subjected to meta-analyses using RevMan 5.3.5 or SAS 9.4. Comparisons between interventions will be performed on one outcome at a time. A risk ratio will be estimated for the studies comparing an intervention group with a usual care or control group. For studies with more than one intervention group, the usual care or a control group is appropriately split for each intervention, and a sensitivity analysis will evaluate the impact of this split $(39,40)$. Any heterogeneity indicated by $\chi 2$ test of heterogeneity and the I2 statistic with its 95\% confidence interval (41) will be investigated through subgroup analyses and the use of a random effects model, when publication bias seems low, according to a funnel plot analysis (42). If interventions have different durations, the effect of the interventions has to be comparable at different times. In such a case, a meta-regression model may be used to investigate the potential effect of intervention duration on the results (43).

\section{Integrated Knowledge Exchange}

The participation of experts in the fields of deprescribing and geriatrics is crucial in order to make the review useful for health professionals and applicable to the Canadian setting. Hence, researchers and KU will meet after the study selection and again before submission of publications of results. Each of these meetings will follow a rigorous and transparent methodology and be documented in detail. They will aim at identifying relevant grey literature and ongoing trials, discussing the selection of studies and answering specific methodological questions. Members will also contribute to the interpretation of review results, giving their own perspective as clinicians, patients and decision makers.

\section{Deriving conclusions and recommendations}

To respond to the first research questions, bottom-line statements based on the evidence gathered or on the results from meta-analyses will be formulated. For the second research question, a matrix will summarize the effective deprescribing interventions, or their components. The results will then be interpreted by the KU team in order to determine knowledge gaps and the need for additional research to validate the identified deprescribing interventions. They will also assist the team in determining which results can be generalized for certain medication groups, and which need further, specific research. Review results will then be modified and finalized through email exchanges.

\section{Discussion}




\section{Expected challenges}

Several challenges may be encountered during the review. Preliminary searches have identified some high-quality deprescribing interventions $(20-23,26,44)$ but there may still be a lack of studies describing health outcomes, as noted by Page et al. (35). Page's literature search was completed in February 2015, so more study results can be expected, given the increased acceptance of deprescribing and the expressed need for more high-quality deprecribing RCT (5). Grey literature was found to be scarce on deprescribing interventions, but trial registers will also be checked to assure complete coverage.

Deprescribing interventions may be available for some medication groups only. We may thus not be able to generalize the evidence on these intervention elements to other medication classes. Given the great variety of studies, it may be difficult to retrieve studies that will be sufficiently homogenous to allow for meta-analyses. Finally, the studies may report various health outcomes and their relevance could be difficult to compare. To solve this problem, the KU experts will evaluate the importance of such limitations, disregarding certain evidence if deemed not relevant for the clinical context.

Deprescribing interventions for different medication classes, such as psychotropics or statins, may yield non-homogeneous results, which will be challenging. There may however exist common characteristics among deprescribing interventions for different medication classes, such as careful patient selection or continuous patient surveillance, leading to positive health outcomes in deprescribing for different medication classes. Finally, different health outcomes may be reported for interventions on similar medications, for instance blood pressure or incidence of cardiovascular disease for the deprescribing of antihypertensives, and it may be challenging to compare their relevance.

\section{Risk of bias}

Selection and information bias

Deprescribing was termed in 2003 (45) and became a Medical Subject Heading (MeSH) term in 2016. Therefore larger, more scoping terms, such as "discontinuation", will be used for database searches regarding earlier studies on medication discontinuation. We expect this to lead to a large number of retrieved references. Having two independent reviewers to screen all references is meant to limit selection bias. The team will carefully verify grey literature to gain a more complete review.

Confounding bias

Retrieved studies may lack homogeneity, making planned meta-analyses more difficult. Moreover, some health outcomes may not be comparable. We will perform narrative syntheses for medication classes where meta-analyses will be impossible. Furthermore, confounding factors may have not been considered in some studies, affecting the quality grades of these studies and of the resulting evidence.

\section{Abbreviations}


PIM

Potentially inappropriate medication

PIMs

Potentially inappropriate medications

RTC

Randomized controlled trials

$\mathrm{KU}$

Knowledge users

RTI

Research Triangle Institute

$\mathrm{MeSH}$

Medical Subject Heading

\section{Declarations}

\section{Ethics approval and consent to participate}

Not applicable.

\section{Consent for publication}

Not applicable.

\section{Availability of data and materials}

All data generated or analysed during this study will be included in published articles.

\section{Competing interests}

The authors declare that they have no competing interests.

\section{Funding}

This work is supported by the Canadian Institute of Health Research (CIHR), grant number [366106].

\section{Authors' contribution}

All authors discussed core ideas, participated in the interpretation of data and contributed to the writing of the paper. All authors read and approved the final manuscript. Edeltraut Kröger is the corresponding author; she will provide full access to all aspects of the research and writing process upon request; she will take final responsibility for the paper. 


\section{Acknowledgements}

The authors wish to thank a number of student interns and research assistants or associates who contributed at different steps of this review (Thuy-An Duong, Dani Abas, Rosa Martins and Norma Perez) to the development of this study protocol or the selection process.

\section{Dissemination plan}

The results of the review will be published in peer-reviewed open-access journals and disseminated to all stakeholders in different forms, e.g. as web-based guidance, as part of continuous education material oras documentation for health professionals. They will be integrated into health professionals' academictraining and presented at appropriate annual meetings andwebsites.Study results, i.e. evidencefor successful interventions and recommendations to adapt or develop interventions will be communicated to patients, caregivers, the scientific community, stakeholders, health professionalsand the general community. An integrated knowledge transfer strategy targets this aim (see table 1).

\section{Bibliography}

1. United Nations DoEaSA, Population Division. World Population Prospects 2019 https://population.un.org/wpp/DataQuery/2009.

2. Masnoon N, Shakib S, Kalisch-Ellett L, Caughey GE. What is polypharmacy? A systematic review of definitions. BMC Geriatr. 2017;17(1):230.

3. Charlesworth CJ, Smit E, Lee DS, Alramadhan F, Odden MC. Polypharmacy Among Adults Aged 65 Years and Older in the United States: 1988-2010. The journals of gerontology Series A, Biological sciences and medical sciences. 2015;70(8):989-95.

4. Wastesson JA-O, Morin LA-O, Laroche ML, Johnell K. How Chronic Is Polypharmacy in Old Age? A Longitudinal Nationwide Cohort Study. (1532-5415 (Electronic)).

5. Frank C, Weir E. Deprescribing for older patients. CMAJ. 2014;186(18):1369-76.

6. Brager R, Sloand E. The spectrum of polypharmacy. The Nurse Practitioner. 2005;30(6):44-50.

7. Fulton MM, Allen ER. Polypharmacy in the elderly: a literature review. J Am Acad Nurse Pract. 2005;17(4):123-32.

8. McLean AJ, Le Couteur DG. Aging biology and geriatric clinical pharmacology. Pharmacological reviews. 2004;56(2):163-84.

9. Endres HG, Kaufmann-Kolle P, Steeb V, Bauer E, Bottner C, Thurmann P. Association between Potentially Inappropriate Medication (PIM) Use and Risk of Hospitalization in Older Adults: An Observational Study Based on Routine Data Comparing PIM Use with Use of PIM Alternatives. PloS one. 2016;11(2):e0146811.

10. American Geriatrics Society 2019 Beers Criteria Update Expert Panel. American Geriatrics Society 2019 Updated AGS Beers Criteria ${ }^{\circledR}$ for Potentially Inappropriate Medication Use in Older Adults. J Am 
Geriatr Soc. 2015;67(4):674-94.

11. Gallagher PF, O'Connor MN, O'Mahony D. Prevention of potentially inappropriate prescribing for elderly patients: a randomized controlled trial using STOPP/START criteria. Clin Pharmacol Ther. 2011;89(6):845-54.

12. Laroche ML, Charmes JP, Merle L. Potentially inappropriate medications in the elderly: a French consensus panel list. Eur J Clin Pharmacol. 2007;63(8):725-31.

13. Renom-Guiteras A, Meyer G, Thurmann PA. The EU(7)-PIM list: a list of potentially inappropriate medications for older people consented by experts from seven European countries. Eur J Clin Pharmacol. 2015;71(7):861-75.

14. Chang CB, Yang SY, Lai HY, Wu RS, Liu HC, Hsu HY, et al. Using published criteria to develop a list of potentially inappropriate medications for elderly patients in Taiwan. Pharmacoepidemiol Drug Saf. 2012;21(12):1269-79.

15. Stuijt CC, Franssen EJ, Egberts AC, Hudson SA. Reliability of the medication appropriateness index in Dutch residential home. Pharm World Sci. 2009;31(3):380-6.

16. Patterson SM, Cadogan CA, Kerse N, Cardwell CR, Bradley MC, Ryan C, et al. Interventions to improve the appropriate use of polypharmacy for older people. Cochrane Database Syst Rev. 2014(10):Cd008165.

17. Patterson S, Hughes C, Kerse N, Cardwell C, Bradley M. Interventions to improve the appropriate use of polypharmacy for older people. Cochrane Database Syst Rev. 2012;5:1-79.

18. Cooper JA, Cadogan CA, Patterson SM, Kerse N, Bradley MC, Ryan C, et al. Interventions to improve the appropriate use of polypharmacy in older people: a Cochrane systematic review. BMJ open. 2015;5(12):e009235.

19. Scott IA, Hilmer SN, Reeve E, Potter K, Le Couteur D, Rigby D, et al. Reducing inappropriate polypharmacy: the process of deprescribing. JAMA internal medicine. 2015;175(5):827-34.

20. Ballard C, Lana MM, Theodoulou M, Douglas S, McShane R, Jacoby R, et al. A randomised, blinded, placebo-controlled trial in dementia patients continuing or stopping neuroleptics (the DART-AD trial). Plos Medicine. 2008;5(4):587-99.

21. Ruths S, Straand J, Nygaard HA, Aarsland D. Stopping antipsychotic drug therapy in demented nursing home patients: a randomized, placebo-controlled study-the Bergen District Nursing Home Study (BEDNURS). Int J Geriatr Psychiatry. 2008;23(9):889-95.

22. Campbell AJ, Robertson MC, Gardner MM, Norton RN, Buchner DM. Psychotropic medication withdrawal and a home-based exercise program to prevent falls: a randomized, controlled trial. J Am Geriatr Soc. 1999;47(7):850-3.

23. Curran HV, Collins R, Fletcher S, Kee SC, Woods B, lliffe S. Older adults and withdrawal from benzodiazepine hypnotics in general practice: effects on cognitive function, sleep, mood and quality of life. Psychol Med. 2003;33(7):1223-37.

24. Kutner JS, Blatchford PJ, Taylor DH Jr, Ritchie CS, Bull JH, Fairclough DL, et al. Safety and benefit of discontinuing statin therapy in the setting of advanced, life-limiting illness: a randomized clinical 
trial. JAMA internal medicine. 2015;175(5):691-700.

25. de Jonge JW, Knottnerus JA, van Zutphen WM, de Bruijne GA, Struijker Boudier HA. Short term effect of withdrawal of diuretic drugs prescribed for ankle oedema. BMJ. 1994;308(6927):511-3.

26. Burr ML, King S, Davies HE, Pathy MS. The effects of discontinuing long-term diuretic therapy in the elderly. Age Ageing. 1977;6(1):38-45.

27. Walma EP, Hoes AW, van Dooren C, Prins A, van der Does E. Withdrawal of long-term diuretic medication in elderly patients: a double blind randomised trial. BMJ. 1997;315(7106):464-8.

28. Farrell B, Pottie K, Thompson W, Boghossian T, Pizzola L, Rashid FJ, et al. Deprescribing proton pump inhibitors: Evidence-based clinical practice guideline. Can Fam Physician. 2017;63(5):354-64.

29. Pottie K, Thompson W, Davies S, Grenier J, Sadowski C, Welch V, et al. Evidence-based clinical practice guideline for deprescribing benzodiazepine receptor agonists. 2016.

30. Bjerre LM, Farrell B, Hogel M, Graham L, Lemay G, McCarthy L, et al. Deprescribing antipsychotics for behavioural and psychological symptoms of dementia and insomnia: Evidence-based clinical practice guideline. Canadian family physician Medecin de famille canadien. 2018;64(1):17-27.

31. Higgins J, Green S, editors. Cochrane Handbook for Systematic Reviews of Interventions: The Cochrane Collaboration; 2011. Available from: .

32. National Institute for Health and Care Excellence. Methods for the development of NICE public health guidance. Third edition ed. London, UK: National Institute for Health and Care Excellence;; 2012. p. 286.

33. Scottish Intercollegiate Guidelines Network (SIGN). SIGN 50: a guideline developer's handbook. SIGN, editor. Edinburgh: SIGN; 2014 October 2014. 53 p.

34. Viswanathan M, Berkman ND. Development of the RTI item bank on risk of bias and precision of observational studies. J Clin Epidemiol. 2012;65:163-78.

35. Page AT, Clifford RM, Potter K, Schwartz D, Etherton-Beer CD. The feasibility and effect of deprescribing in older adults on mortality and health: a systematic review and meta-analysis. $\mathrm{Br} \mathrm{J}$ Clin Pharmacol. 2016;82(3):583-623.

36. Popay J, Roberts H, Sowden A, Petticrew M, Arai Y, Rodgers M, et al. Guidance on the Conduct of Narrative Synthesis in Systematic Reviews. 1 ed. Lancaster, UK: Institute for Health Research, Bowland Tower East Wing, Lancaster University; 2006 April 2006. 92 p.

37. Rodgers M, Sowden A, Petticrew M, Arai L, Roberts H, Britten N, et al. Testing Methodological Guidance on the Conduct of Narrative Synthesis in Systematic Reviews: Effectiveness of Interventions to Promote Smoke Alarm Ownership and Function. Evaluation. 2009;15(1):49-74.

38. Ragin CC. The Comparative Method: Moving Beyond Qualitative and Quantitative Strategies. In: Press UoC, editor. Berkeley 1987.

39. Hedges LV. Research synthesis: the state of the art. Int J Aging Hum Dev. 1984;19(2):85-93.

40. DerSimonian R, Laird N. Meta-analysis in clinical trials. Controlled clinical trials. 1986;7(3):177-88. 
41. Higgins JP, Thompson SG. Quantifying heterogeneity in a meta-analysis. Stat Med. 2002;21(11):1539-58.

42. Borenstein M, Hedges LV, Higgins JP, Rothstein HR. Publication Bias. In: Wiley J, editor Introduction to Meta-Analysis2009.

43. Berkey CS, Hoaglin DC, Mosteller F, Colditz GA. A random-effects regression model for meta-analysis. Stat Med. 1995;14(4):395-411.

44. Garfinkel D, Mangin D. Feasibility study of a systematic approach for discontinuation of multiple medications in older adults: addressing polypharmacy. Arch Intern Med. 2010;170(18):1648-54.

45. Woodward MC. Deprescribing. Achieving Better Health Outcomes for Older People Through Reducing Medications. J Pharm Pract res. 2003;33:323-8.

\section{Tables}

Table 1

End of project knowledge transfer strategy

\begin{tabular}{|c|c|}
\hline Medium & Target \\
\hline $\begin{array}{l}\text { Journal } \\
\text { publication }\end{array}$ & Peer-review open-access journal (undefined yet) \\
\hline Communication & $\begin{array}{l}\text { Annual meeting of: } \\
\text { - Canadian Geriatrics Society } \\
\text { - Canadian Association of Population Therapeutics } \\
\text { - Family Medicine Forum } \\
\text { - FRQS's, the Quebec Network for Research on Aging } \\
\text { - Canadian Pharmacists' Association (CPhA) }\end{array}$ \\
\hline $\begin{array}{l}\text { Website } \\
\text { publication }\end{array}$ & $\begin{array}{l}\text { Publishing the results on the follow websites: } \\
\text { - Canadian Geriatrics Society } \\
\text { - Canadian Association of Population Therapeutics } \\
\text { - Family Medicine Forum } \\
\text { - FRQS's, the Quebec Network for Research on Aging }\end{array}$ \\
\hline $\begin{array}{l}\text { Web based } \\
\text { guidance and } \\
\text { guidance's } \\
\text { documents }\end{array}$ & $\begin{array}{l}\text { Present successful characteristics of deprescribing intervention on Canadian } \\
\text { Pharmacists' Association (CPhA) website via the initiative "the Translator". } \\
\text { The Quebec "Institute National d'Excellence en Santé et en Services Sociaux } \\
\text { (INESSS)" will participate in knowledge exchange via the development of } \\
\text { guidance documents. }\end{array}$ \\
\hline $\begin{array}{l}\text { Education and } \\
\text { training }\end{array}$ & $\begin{array}{l}\text { Offer a continuous education activity for health professionals, led by the The } \\
\text { Centre d'excellence sur le vieillissement de Québec (CEVQ) which has a strong } \\
\text { record of continuous education for clinicians in all settings. } \\
\text { Integrate into health professionals' academic training. }\end{array}$ \\
\hline $\begin{array}{l}\text { Continuous } \\
\text { collaborations }\end{array}$ & $\begin{array}{l}\text { Pursue our collaboration with several institutions: } \\
\text { - Seniors Health Research Transfer Network and OPEN (Ontario) } \\
\text { - Institute for Health Services and Policy Research at the Canadian Institutes of } \\
\text { Health Research } \\
\text { - Canadian Dementia Knowledge Translation Network }\end{array}$ \\
\hline
\end{tabular}




\section{Supplementary Files}

This is a list of supplementary files associated with this preprint. Click to download.

- PRISMAPchecklist30juinJL.docx

- Annexesdeprotocoledeprescribing1juilletJL.docx 